\title{
The enhancement of pericentromeric cohesin association by conserved kinetochore components promotes high-fidelity chromosome segregation and is sensitive to microtubule-based tension
}

\author{
Carrie A. Eckert, ${ }^{1,2}$ Daniel J. Gravdahl, ${ }^{2}$ and Paul C. Megee ${ }^{1,2,3}$ \\ ${ }^{1}$ Program in Molecular Biology, University of Colorado Health Sciences Center, Aurora, Colorado 80045, USA; ${ }^{2}$ Department \\ of Biochemistry and Molecular Genetics, University of Colorado Health Sciences Center, Aurora, Colorado 80045, USA
}

\begin{abstract}
Sister chromatid cohesion, conferred by the evolutionarily conserved cohesin complex, is essential for proper chromosome segregation. Cohesin binds to discrete sites along chromosome arms, and is especially enriched surrounding centromeres, but past studies have not clearly defined the roles of arm and pericentromeric cohesion in chromosome segregation. To address this issue, we developed a technique that specifically reduced pericentromeric cohesin association on a single chromosome without affecting arm cohesin binding. Under these conditions, we observed more extensive stretching of centromeric chromatin and elevated frequencies of chromosome loss, suggesting that pericentromeric cohesin enrichment is essential for high-fidelity chromosome transmission. The magnitude of pericentromeric cohesin association was negatively correlated with tension between sister kinetochores, with the highest levels of association in cells lacking kinetochore-microtubule attachments. Pericentromeric cohesin recruitment required evolutionarily conserved components of the inner and central kinetochore. Together, these observations suggest that pericentromeric cohesin levels reflect the balance of opposing forces: the kinetochore-mediated enhancement of cohesin binding and the disruption of binding by mechanical tension at kinetochores. The involvement of conserved kinetochore components suggests that this pathway for pericentromeric cohesin enrichment may have been retained in higher eukaryotes to promote chromosome biorientation and accurate sister chromatid segregation.
\end{abstract}

[Keywords: Chromosome biorientation; sister chromatid cohesion; cohesin; kinetochore; mitosis; genomic integrity]

Supplemental material is available at http://www.genesdev.org.

Received October 2, 2006; revised version accepted December 5, 2006.

To segregate correctly in mitosis, replicated sister chromatids must form stable attachments to microtubules that emanate from opposite spindle poles of the dividing cell, a process referred to as chromosome biorientation. Attachments to chromosomes are mediated by the kinetochore, an elaborate protein complex that assembles within centromeric DNA and mediates the capture of a single or multiple microtubules depending on the complexity of the kinetochore (Bloom 1993). Kinetochoremicrotubule capture is stochastic, resulting in chromo-

${ }^{3}$ Corresponding author.

E-MAIL paul.megee@uchsc.edu; FAX (303) 724-3215.

Article published online ahead of print. Article and publication date are online at http://www.genesdev.org/cgi/doi/10.1101/gad.1498707. somes that are initially attached to only one pole (monooriented). Two activities are then thought to promote attachment of unoccupied sister kinetochores to microtubules from opposite poles. First, dynamic instability of kinetochore microtubules produces oscillatory chromosome movements that increase the probability of microtubule capture by the unattached kinetochore (Skibbens et al. 1993). In addition, the "sliding" of mono-oriented chromosomes toward the spindle mid-zone along the kinetochore microtubules of a neighboring bioriented chromosome may also favor the capture of microtubules originating from the opposite pole (Kapoor et al. 2006). The arbitrary nature of these events may result in the formation of syntelic attachments, where both kinetochores in a sister chromatid pair are attached to micro- 
tubules from the same pole. The failure to eliminate syntelic attachments prior to sister chromatid separation may lead to the transmission of abnormal chromosome numbers to daughter cells. This condition, known as aneuploidy, often results in genetic disease or miscarriage and is also a hallmark of tumor cells. Thus, mechanisms that promote chromosome biorientation and correct aberrant microtubule attachments are essential for the prevention of genomic instability.

The robust association, or cohesion, of sister chromatids in centromeric regions is likely to promote chromosome biorientation, possibly by constraining the microtubule-binding sites on sister kinetochore pairs in opposite directions. This arrangement theoretically favors the capture of microtubules emanating from opposite poles. In support of this model, the elimination of cohesion in budding yeast increases syntelic attachments (Tanaka et al. 2000). Sister chromatid cohesion is also instrumental for the generation of tension between sisters, which is thought to play important roles in stabilizing amphitelic (bipolar) attachments and also in resisting poleward microtubule forces until all chromosomes have achieved biorientation (Nicklas and Ward 1994). Interestingly, the tension exerted on bioriented chromosomes is capable of separating sister chromatids in centromere-proximal regions despite robust centromeric cohesion (Waters et al. 1996; Goshima and Yanagida 2000; He et al. 2000; Tanaka et al. 2000; Sonoda et al. 2001). However, this preanaphase separation is transient, as sister chromatid centromeric regions frequently reestablish associations prior to anaphase onset.

Sister chromatid cohesion is mediated by a multisubunit complex, called cohesin, whose constituents have been conserved in organisms spanning the yeasts to vertebrates (Nasmyth and Haering 2005). Cohesin is composed of four subunits: two members of the SMC family of chromosomal ATPases (Smc1 and Smc3), as well as two non-SMC subunits (Scc3 and Mcd1/Scc1). Biophysical studies have shown that the cohesin subunits form a ring-shaped complex, leading to the suggestion that cohesins may topologically encircle sister chromatids (Haering et al. 2002; Gruber et al. 2003; Ivanov and Nasmyth 2005). However, the precise mechanism by which cohesin rings interact with chromatin to mediate cohesion remains unknown (Huang et al. 2005). Genomewide maps of cohesin-binding sites have also contributed to our understanding of sister chromatid cohesion. Cohesin and Pds5, another mediator of cohesion, associate with discrete sites along budding and fission yeast chromosomes (Blat and Kleckner 1999; Hartman et al. 2000; Laloraya et al. 2000; Glynn et al. 2004; Lengronne et al. 2004). These sites are frequently $\mathrm{A}+\mathrm{T}$-rich, and are located between convergently transcribed genes. Notably, cohesin association is especially enriched in pericentromeric regions from $\mathrm{S}$ phase through metaphase in mitosis (Blat and Kleckner 1999; Megee et al. 1999; Glynn et al. 2004; Lengronne et al. 2004; Weber et al. 2004). Although high-resolution mapping of cohesins in higher eukaryotes is not yet available, cytological evidence suggests that the pericentromeric enrichment of cohesins is characteristic of higher eukaryotes as well (Fukagawa et al. 2004).

Although several factors that mediate cohesin loading have been identified, the mechanism of cohesin recruitment to specific genomic locations is not well understood. Recent advances suggest that histone post-translational modifications mediate cohesin recruitment to sites of DNA damage and also to the pericentromeric domains of regional centromeres. For example, cohesin's interaction with phosphorylated histone H2A $(\gamma$-H2AX in metazoans), which accumulates throughout broad regions flanking a DNA double-strand break, is responsible for its recruitment to sites of DNA damage (Ünal et al. 2004). Furthermore, the association of cohesin with Swi6, the Schizosaccharomyces pombe ortholog of heterochromatin protein 1 (HP1), contributes to cohesin recruitment to pericentromeric heterochromatin in fission yeast (Bernard et al. 2001; Nonaka et al. 2002). Swi6 is essential for heterochromatin formation, and is recruited to centromeric regions by the binding of its chromodomain to methylated Lys 9 residues of histone $\mathrm{H} 3$, a modification commonly found in heterochromatin. Budding yeast pericentromeric cohesin enrichment must occur through an alternative mechanism, however, given that this organism lacks an HP1 homolog, H3 Lys 9 methylation, and repetitive heterochromatic DNA flanking centromeres. Accordingly, we have recently demonstrated that the budding yeast centromere/kinetochore complex functions as an enhancer of cohesin recruitment, generating large $\sim 20$ - to $50-\mathrm{kb}$ pericentromeric domains that are highly enriched for cohesin binding (Weber et al. 2004). Whether the kinetochore-mediated enhancement of cohesin association in budding yeast also involves histone post-translational modifications remains to be determined.

The apparent conservation of the pericentromeric cohesin enrichment in eukaryotes and the possible existence of multiple pathways for pericentromeric cohesin recruitment suggest that this enrichment plays an important role in chromosome segregation and the maintenance of genomic integrity. To distinguish between the relative contributions of arm and pericentromeric cohesion in chromosome segregation, we have specifically reduced pericentromeric cohesin association on a single chromosome without affecting arm cohesion, which had not been possible using previous experimental approaches. We find that chromosomes with reduced pericentromeric cohesin association exhibit elevated levels of pericentromeric sister chromatid separation and chromosome loss. Using a collection of conditional kinetochore mutants, we have found that conserved kinetochore subunits mediate enhanced cohesin association in pericentromeric regions, suggesting that a kinetochoredirected pathway for pericentromeric cohesin recruitment may be retained in higher eukaryotes. Lastly, we provide evidence that microtubule-based tension at kinetochores reduces pericentromeric cohesin association, indicating that chromosome biorientation is accompanied by a modification of cohesin association in these regions. 
Eckert et al.

\section{Results \\ Disruption of pericentromeric cohesin enrichment affects centromere separation and chromosome transmission fidelity}

To directly assess the importance of pericentromeric cohesion relative to arm cohesion on the fidelity of chromosome segregation, we have flanked the centromere on chromosome III (CHRIII) with "insulator" sequences that reduce cohesin association throughout $\sim 40 \mathrm{~kb}$ spanning CEN3. Briefly, an $\sim 4.5$-kb fragment of $\mathrm{G}+\mathrm{C}$-rich $(\sim 64 \%)$ Myxococcus DNA and an $\sim 0.9-\mathrm{kb}$ intergenic region containing the budding yeast $P G K 1$ promoter were inserted to the left and right, respectively, of CEN3 (hereafter referred to as I-CEN3-I). The mechanism(s) whereby these sequences limit pericentromeric cohesin binding is unclear. The high G $+\mathrm{C}$ content of Myxococcus DNA may be unfavorable for cohesin association, which is biased toward regions with high $\mathrm{A}+\mathrm{T}$ base composition (Blat and Kleckner 1999; Megee et al. 1999; Glynn et al. 2004). Similarly, the PGK1 promoter (CHRIII, $\sim 137 \mathrm{~kb}$ ) was tested for its ability to affect cohesin association, as this region is consistently devoid of cohesin binding while flanking sequences exhibit avid cohesin association (Weber et al. 2004). The inability of cohesins to associate with the $P G K 1$ promoter region may be linked to its strong transcriptional activity, as transcription is incompatible with cohesin binding (Glynn et al. 2004; Lengronne et al. 2004).

To verify that these sequences indeed reduced cohesin association in centromere-flanking regions, we examined the distribution and magnitude of cohesin binding by chromatin immunoprecipitation (ChIP) using epitope-tagged Mcd1 (MCD1-6HA) as a marker for the cohesin complex. DNA cross-linked to Mcdl as well as genomic DNA not subjected to immunoprecipitation was isolated and analyzed by PCR in reactions that responded linearly to the amount of input DNA. Unless otherwise indicated, cultures used for ChIP analyses were first synchronized in the G1 period of the cell cycle using $\alpha$-factor mating pheromone, and then released from the G1 arrest into fresh media at the appropriate temperature. Cells were then cross-linked when populations were highly enriched for preanaphase cells. This enrichment was achieved using one of three methods: (1) treatment with the microtubule poison, nocodazole (NZ); (2) a conditional mutant in the anaphase-promoting complex (APC, see below); or (3) a microscopic analysis of cell morphology.

Isogenic wild-type and I-CEN3-I cells were released from G1 and arrested in mitosis using NZ and then processed for ChIP. We observed that the levels of pericentromeric sequences cross-linked to Mcd1 were reduced throughout an $\sim 40-\mathrm{kb}$ domain spanning CEN3 in the I-CEN3-I strain when compared with wild-type cells (Fig. 1A). Interestingly, the region exhibiting this reduction in Mcdl association corresponded well with the centromeric region whose cohesin enrichment was shown previously to be dependent on the kinetochore (Weber et al. 2004). In contrast, Mcdl association with CHRIII arm
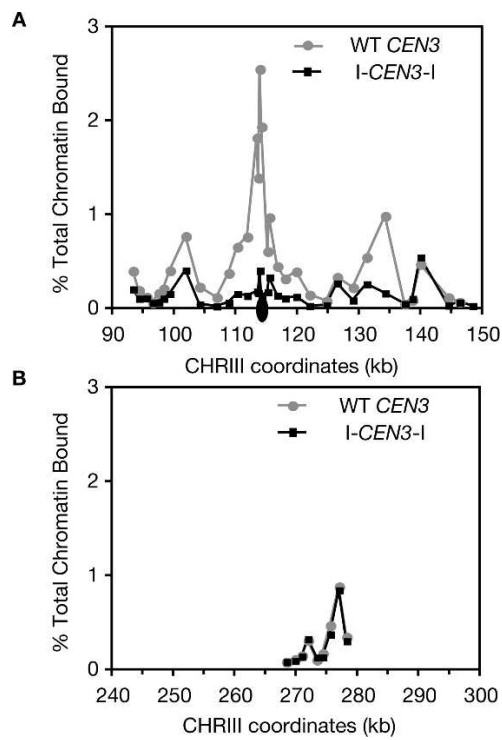

Figure 1. Pericentromeric cohesin enrichment is prevented by centromere-flanking "insulators." Wild-type (CEY70) and I-CEN3-I (CEY40) cells containing Mcd1-6HA were released from G1 arrest into media containing $15 \mu \mathrm{g} / \mathrm{mL}$ NZ. Cultures were processed for ChIP when $>90 \%$ large budded, indicating metaphase arrest. ChIP was performed using HA antiserum. DNA cross-linked to Mcdl and diluted input DNA not subjected to immunoprecipitation were analyzed by PCR using primer pairs that amplify $\sim 300$-bp fragments within the CHRIII pericentromeric region $(A)$ or at a centromere-distal CHRIII region $(B)$. Arm and pericentromeric Mcd1 binding data are plotted on equivalent scales for comparison. Quantitation of DNA in the Mcd1 ChIPs, expressed as a percentage of the input DNA, is plotted as a function of the locations of the midpoints of those DNA fragments based on the Saccharomyces Genome Database (SGD) coordinates. The position of the centromere is indicated by a black oval.

sequences was equivalent in the two strains (Fig. 1B). These observations suggest that the insulator sequences specifically diminish kinetochore enhancer activity for cohesin recruitment, resulting in a significant reduction in pericentromeric, but not arm, cohesin association.

To investigate the effects of reduced pericentromeric cohesin binding on chromosome segregation, sister chromatids were visualized by the binding of GFP-Lac repressor fusion protein to oligomerized Lac operators inserted to the left of $C E N 3, \sim 19 \mathrm{~kb}$ away on wild-type CHRIII or $\sim 23 \mathrm{~kb}$ away in cells containing I-CEN3-I (due to the insertion of $\sim 4.5 \mathrm{~kb}$ Myxococcus DNA) (Fig. 2). Poleward microtubule-dependent forces are responsible for the transient separation of sister chromatids throughout an $\sim 20$-kb pericentromeric region, or $\sim 10 \mathrm{~kb}$ on either side of the centromere (He et al. 2000). Thus, the operators in our strains are inserted beyond the region that normally exhibits sister chromatid separation. Isogenic wild-type and I-CEN3-I cells were first staged in G1, and then released from the arrest into fresh media. Sister chromatid cohesion and spindle microtubules were examined in separate aliquots of cells withdrawn prior to release and at 15-min intervals post-release. Spindle elongation, an 

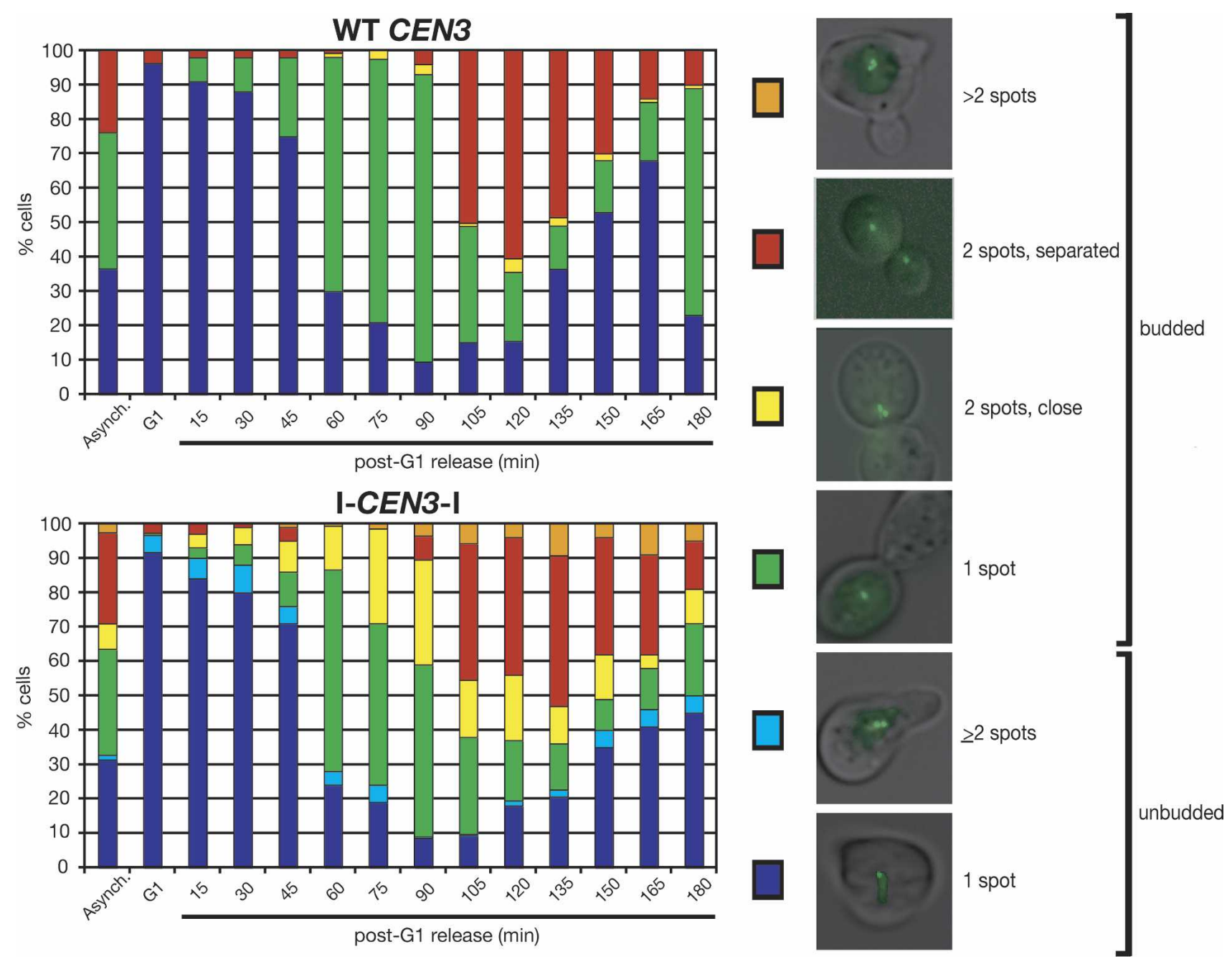

Figure 2. Disruption of pericentromeric cohesin enrichment extends the domain subject to stretching. LacOp arrays were placed to the left of CEN3, $19 \mathrm{~kb}$ away on wild-type (CEY90) or $\sim 23 \mathrm{~kb}$ away in I-CEN3-I (CEY91) cells (at SGD coordinate 95678) that express a LacI-GFP fusion protein. Cells were staged in G1 with $\alpha$-factor and released in fresh medium lacking pheromone. Aliquots of cells were collected for an asynchronous control, G1-arrested cells, and at 15-min intervals post-release, and fixed in paraformaldehyde. Bud morphology and the number and position of GFP spots were scored for each time point $(n \geq 100)$.

indicator of anaphase onset, began at $\sim 105 \mathrm{~min}$ in both wild-type and I-CEN3-I cultures (Supplementary Fig. 1A). The appearance of budded wild-type cells with two GFP spots increased from $\leq 5 \%$ at $90 \mathrm{~min}$ to $50 \%$ at 105 min, which corresponded well with the timing of spindle elongation, suggesting that the resolution of the GFP signal into two spots is due to sister chromatid segregation in these cells. In contrast, I-CEN3-I cultures contained $\geq 25 \%$ budded cells with two closely apposed GFP spots as early as $75 \mathrm{~min}$ post-release (Fig. 2, yellow), $\sim 20 \mathrm{~min}$ prior to the initiation of spindle elongation. Furthermore, cells with multiple GFP spots appeared at late time course intervals in I-CEN3-I, but not wild-type, cells (Fig. 2, orange), suggesting that chromosome missegregation occurred in a small fraction of cells containing the insulator-flanked kinetochore. Taken together, these observations suggest that the stretching of centromeric chromatin is more extensive when pericentro- meric cohesin binding is limited and may contribute to chromosome missegregation.

Several observations suggest that the increased incidence of multiple GFP spots is unlikely to be due to aneuploidy caused solely by a kinetochore defect in I-CEN3-I cells. Fewer than 5\% of G1-staged I-CEN3-I cells exhibited multiple GFP spots (Fig. 2, light blue), and this proportion remained unchanged in newly formed daughter cells produced at late time course intervals, suggesting that the kinetochore is functional in I-CEN3-I cells. In contrast, $\sim 25 \%$ of G1-staged wild-type CEN3 or I-CEN3-I cells containing a deletion of CTF19, a nonessential gene encoding a kinetochore subunit, have multiple GFP spots, indicative of chromosome missegregation in a previous cell division cycle (Supplementary Fig. 2). Furthermore, we observed no increase in the generation time of I-CEN3-I cells, or sensitivity to the microtubule poison, benomyl, either in the presence or ab- 
sence of the spindle checkpoint gene, MAD2 (Supplementary Fig. 1B). These phenotypes are characteristic of kinetochore mutants, such as ctf19s (Supplementary Fig. 1B), and would be expected if chromosome missegregation were pervasive in I-CEN3-I cells due to the presence of a defective kinetochore. Lastly, preanaphase I-CEN3-I cells with Lac operators inserted $\sim 37 \mathrm{~kb}$ to the right of CEN3 had single GFP spots, indicating that cohesion in more centromere-distal regions was unaffected by insulator insertion (Fig. 1; data not shown). Taken together, these observations strongly suggest that the insulator-flanked kinetochore does not adversely affect kinetochore function.

To more accurately determine whether limiting pericentromeric cohesin association increases chromosome missegregation, we measured mitotic chromosome loss in a diploid containing a wild-type MAT $\alpha$ CHRIII homolog and a MATa CHRIII homolog with an insulatorflanked centromere (Fig. 3A). In haploids, a or $\alpha$ matingtype information resides at the MAT locus on CHRIII. Diploids express both $\mathbf{a}$ and $\alpha$ information and are normally sterile, but can mate if the strain becomes monosomic for CHRIII due to chromosome loss or a gene conversion event at $M A T$, resulting in a/a or $\alpha / \alpha$ diploids. To distinguish between chromosome loss and gene conversion events, the URA3 gene was inserted $\sim 100 \mathrm{~kb}$ from the MAT locus on the MATa homolog in the diploids.

Wild-type haploids mated with efficiencies of $\sim 20 \%$ in quantitative assays, whereas wild-type diploid cells crossed to haploids of either mating type failed to mate, having efficiencies of $<0.0001 \%$ (Fig. 3B). Furthermore, a

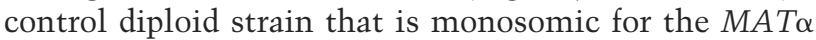
CHRIII homolog mated as efficiently as a MAT $\alpha$ haploid. In contrast, the I-CEN3-I heterozygous diploid mated with an efficiency of $\sim 0.2 \%$ when crossed to a MATa haploid, a 4200-fold increase compared with the isogenic wild-type diploid. Importantly, $<0.25 \%$ of the resultant triploid strains were uracil prototrophs, indicating that chromosome loss and not gene conversion of the MAT locus was responsible for the elevated mating proficiency in the insulator-flanked diploid (data not shown). Notably, the I-CEN3-I heterozygous diploid failed to mate efficiently when crossed to a MATa haploid (Fig. $3 \mathrm{~B})$. This preferential mating of the I-CEN3-I diploid strain with a MATa haploid suggests that the MATa homolog, which contains I-CEN3-I, is lost selectively at high frequency. Taken together, these observations suggest that while arm cohesion may partially compensate for the absence of robust centromeric cohesion, the loss of centromeric cohesion indeed results in large increases in chromosome loss.

Pericentromeric cohesin binding is elevated in the absence of kinetochore-microtubule attachments

To further investigate the nature of pericentromeric cohesin enrichment, we examined Mcd1 binding in the pericentromeric regions of cells arrested in mitosis using a conditional $c d c 16$ mutant, in either the presence or absence of NZ. CDC16 encodes a subunit of the APC, and $c d c 16$ mutant cells grown at the restrictive temperature arrest in metaphase due to the inability to degrade Pds1, a negative regulator of anaphase (Yamamoto et al. 1996; Cohen-Fix and Koshland 1997). The APC is also disabled in NZ-treated cells due to the activation of the spindle assembly checkpoint pathway. This surveillance pathway detects aberrant attachments, and may delay anaphase onset to prevent mitotic catastrophe. Whether the checkpoint is activated by the presence of unoccupied kinetochore-microtubule-binding sites, or by the absence of tension between sister kinetochores that accompanies aberrant attachments remains enigmatic (Pinsky and Biggins 2005). Nevertheless, the establishment of stable amphitelic attachments satisfies the
Figure 3. Disruption of pericentromeric cohesin enrichment decreases chromosome transmission fidelity. (A) A schematic of the MATa I-CEN3-I/MAT $\alpha$ wild-type CEN3 diploid is shown. CEN3 is indicated by the oval, and the $\mathrm{G}+$ C-rich Myxococcus DNA and the $P G K 1$ region flanking the centromere are shown as black and white boxes, respectively. URA3 is integrated near the telomere on the MATa homolog. (B) Chromosome loss was determined using quantitative mating assays (Materials and Methods). A haploid MAT $\alpha$ strain and diploids that were either monosomic for MAT $\alpha$ CHRIII (CEY102) or contained a MATa copy marked with URA3 for selection and either wild-type (WT) CEN3 (CEY39) or I-CEN3-I (CEY61) were crossed to $M A T \mathbf{a}$ and MAT $\alpha$ haploid testers to determine their ability to mate. Mating due to loss of the CHRIII MATa homolog, as opposed to gene conversion of the MAT locus, was determined by growth on minimal media lacking uracil.

\section{A}

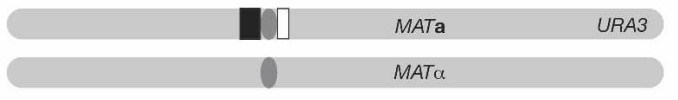

B

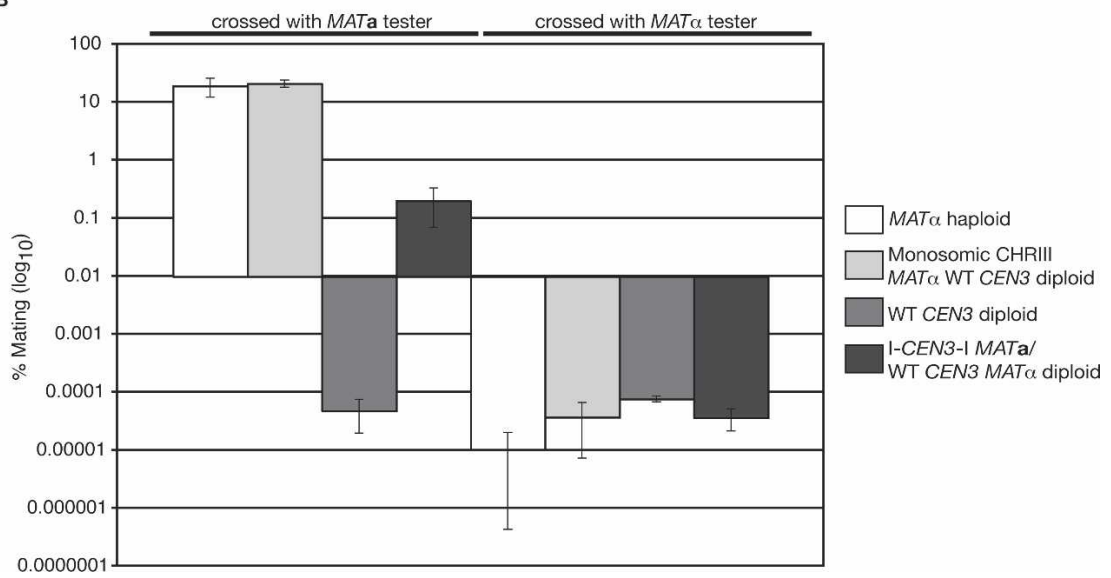


checkpoint and removes the block to cell cycle progression. Thus, NZ treatment and the conditional cdc16 mutation both arrest cells in metaphase due to the inactivation of the APC.

In this experiment, $c d c 16$ cells were released from a G1 arrest into fresh media at the restrictive temperature containing either NZ or DMSO as a vehicle control, and then processed for ChIP when arrested in metaphase. We observed that the levels of sequences corresponding to chromosome arm cohesin-associated regions were similar in the Mcdl ChIPs under the two mitotic arrest conditions (Fig. 4A). In contrast, the magnitude of pericentromeric sequences was approximately threefold higher in the ChIPs prepared from NZ-treated cdc16 cells when compared with those obtained from cells arrested by a cdc16 mutation alone (Fig. 4B). Thus, these observations indicate that mitotic arrest per se does not result in elevated levels of pericentromeric cohesin binding.

The spindle assembly checkpoint is neither necessary nor sufficient for increased pericentromeric cohesin association

While the conditional $c d c 16$ mutation and NZ treatment both lead to APC inactivation, other aspects of the two mitotic arrests are different. The absence of kinetochore-microtubule attachments in NZ-treated cells leads to an activated checkpoint, while the conditional $c d c 16$ mutant arrests in mitosis with normal amphitelic attachments and an inactive spindle checkpoint (Hardwick et al. 1999). Thus, the higher magnitude of pericentromeric cohesin binding in NZ-treated cells may be mediated directly by the spindle assembly checkpoint, or alternatively, it could be due specifically to alterations in kinetochore-microtubule interactions.

To distinguish between these two possibilities, we first examined the magnitude of pericentromeric Mcd1 binding in cells that overexpress MPS1, which encodes a spindle assembly checkpoint component. MPS1 overexpression imposes a checkpoint-mediated mitotic arrest that is independent of spindle damage (Hardwick et al. 1996). Thus, this approach allowed us to separate the effects of checkpoint activation and the loss of kinetochore-microtubule attachments on pericentromeric cohesin association. Cells containing a galactose-inducible MPS1 gene were staged in G1 and then released into NZor DMSO-containing media in the presence or absence of galactose. Control cells lacking NZ treatment or MPS1 overexpression (i.e., DMSO only) do not arrest in metaphase, and were therefore monitored microscopically upon release from G1 arrest to enrich for preanaphase cells (as confirmed by flow cytometry; data not shown).

The levels of CHRIII pericentromeric sequences present in Mcdl ChIPs in cells with and without MPS1 overexpression were similar, and were on average approximately threefold lower than those observed in NZtreated cells (Fig. 4C). Thus, checkpoint activation per se does not result in increased pericentromeric cohesin binding. In addition, the levels of pericentromeric sequences in Mcdl ChIPs prepared from NZ-treated cells
A

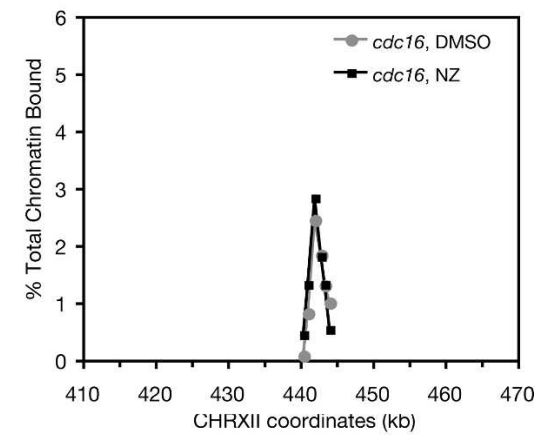

B

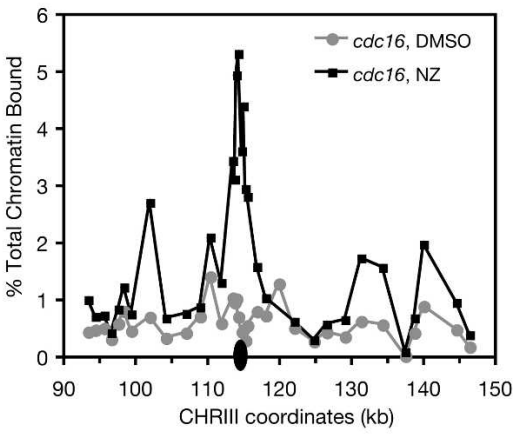

C

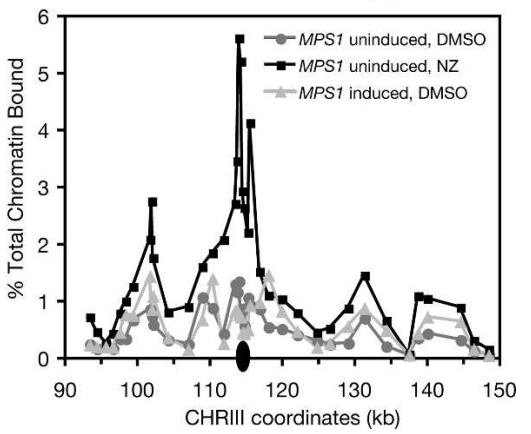

Figure 4. Mcdlp binding in the CEN3 pericentromeric region increases in the absence of microtubules and is independent of the spindle assembly checkpoint. (A) Mcd1-6HA $c d c 16$ cells (1829-15B) were released from G1 arrest into $37^{\circ} \mathrm{C}$ prewarmed media containing either $15 \mu \mathrm{g} / \mathrm{mL} \mathrm{NZ}$ or DMSO until arrested $(\sim 3 \mathrm{~h})$, as determined by large-budded cell morphologies. Cells were processed for ChIP using HA antibody. DNA cross-linked to Mcd1 and diluted input DNA were analyzed as described in Figure 1 for a CHRXII arm region $(A)$ and the CHRIII pericentromeric region $(B) .(C)$ Mcd1-6HA pGAL-MPS1 cells (PMY319) were staged in $\mathrm{G} 1$ in rich media containing raffinose $(2 \%$ final concentration). Cells were released into NZ- or DMSO-containing media in the presence or absence of galactose $4 \%$ final concentration) to induce Mpslp overexpression. Cells were processed for ChIP when mostly large budded, and cell samples were collected for flow-cytometric analyses to confirm metaphase enrichment (data not shown). The quantitation of CHRIII pericentromeric DNA in Mcd1 ChIPs was done as described in the legend for Figure 1.

were similar with or without MPS1 overexpression, indicating that MPS1 overexpression had no additive effect on the magnitude of cohesin binding in pericentromeric regions (data not shown). Lastly, cdc16 mutant cells containing a deletion of spindle checkpoint genes BUB1 or $M A D 2$ responded to NZ treatment with increased peri- 
centromeric Mcd1 association at levels similar to those observed in an isogenic cdc16 mutant control (Supplementary Fig. 3; data not shown). Taken together, these observations indicate that the spindle assembly checkpoint is neither necessary nor sufficient for increased pericentromeric cohesin binding in response to the loss of kinetochore-microtubule attachments.

\section{Decreased tension across sister kinetochores results in increased pericentromeric cohesin binding}

We next tested whether the different magnitudes of pericentromeric cohesin binding observed in cdc16 cells in the presence or absence of microtubules reflected the level of mechanical tension across sister kinetochore pairs. To address this possibility, we examined pericentromeric Mcd1 association in a variety of conditional kinetochore mutants that disrupt force generation at kinetochores by altering kinetochore-microtubule interactions. Mutant phenotypes observed in this collection include the complete detachment of chromosomes from microtubules, varying degrees of syntelic attachments, and those with amphitelic attachments with reduced tension. If mechanical tension derived from amphitelic attachments normally reduces pericentromeric cohesin levels, we expected the conditional kinetochore mutants to instead have elevated levels of pericentromeric cohesin association.

We first analyzed pericentromeric Mcd1 binding upon complete kinetochore-microtubule detachment, conditions that mimic NZ treatment. NDC80 encodes a subunit of the conserved central kinetochore Ndc80 complex, which is essential for kinetochore-microtubule attachment (He et al. 2001; Janke et al. 2001; Wigge and Kilmartin 2001). At the restrictive temperature, ndc80-1 cells fail to recruit outer kinetochore components and chromosomes detach from the spindle (He et al. 2001). Despite being spindle checkpoint-proficient, ndc80-1 cells undergo spindle elongation (He et al. 2001; McCleland et al. 2003). Therefore, we analyzed ndc80-1 in a cdc16 mutant background to impose a uniform metaphase arrest. Interestingly, we found that ndc80-1 cdc16 cells had threefold higher levels of sequences corresponding to an $\sim 20$-kb CEN3-spanning region in Mcd1 ChIPs when compared with the levels seen in isogenic cdc16 cells, which retain bioriented chromosomes (Fig. $5 \mathrm{~A})$. However, the levels of pericentromeric sequences in Mcd1 ChIPs prepared from ndc80-1 cdc16 cells without NZ were indistinguishable from those obtained in the presence of NZ (Fig. 5A). Thus, we conclude that elevated levels of pericentromeric cohesin association are characteristic of cells that lack tension, due to the absence of either functional kinetochores (ndc80-1) or microtubules (NZ treatment).

We next analyzed several mutants of the outer kinetochore that have increased levels of syntelic microtubule attachments, which fail to produce tension as both kinetochores attach to one pole. Recent observations indicate that Ipl1, the budding yeast Aurora B kinase, is an active participant in the spindle checkpoint, where it likely senses the absence of tension resulting from aberrant kinetochore-microtubule interactions (Biggins et al. 1999; Kim et al. 1999; Biggins and Murray 2001; Tanaka et al. 2002; Pinsky et al. 2006). In addition, the Ipl1 kinase also destabilizes syntelic microtubule interactions, thereby allowing additional opportunities to form amphitelic attachments (Biggins et al. 1999; Tanaka et al. 2002). Targets of the Ipll kinase include two subunits of the DASH complex, which associates with kinetochores in a microtubule-dependent manner (Cheeseman et al. 2001a, 2002; Janke et al. 2002; Li et al. 2002; Miranda et al. 2005). Mcd1 ChIPs of ipl1-321 cells, performed in a cdc16 background to provide a uniform mitotic arrest, revealed that the levels of pericentromeric sequences from a 20-kb CEN3-spanning region were on average fourfold higher than isogenic control cdc16 cells (Fig. $5 \mathrm{~B})$, while equivalent levels of CHRIII pericentromeric sequences were present in Mcdl ChIPs prepared from double- and single-mutant cells treated with NZ. Importantly, syntelic attachments produced in conditional mutants of DASH complex subunits, dam1-11 and dad11 , were shown to result in varying degrees of chromosome missegregation, namely $85 \%-99 \%$ of dam $1-11$ cells and $\sim 30 \%$ of dad1-1 cells (Cheeseman et al. 2001b; Enquist-Newman et al. 2001; Janke et al. 2002; Li et al. 2002). Thus, it was striking to observe a hierarchy of pericentromeric cohesin levels in this experiment: The dam1-11 mutant, which has the more penetrant chromosome missegregation phenotype, had higher levels of CHRIII pericentromeric sequences in Mcdl ChIPs than did the dad1-1 mutant (Supplementary Fig. 4A,B), and both mutants had higher levels of CHRIII pericentromeric levels in Mcd1 ChIPs than did the isogenic wildtype control (Supplementary Fig. 4B). Thus, these results suggest that the analysis of pericentromeric cohesin binding by ChIP provides a sensitive measure of the levels of tension within pericentromeric chromatin.

Lastly, we analyzed pericentromeric cohesin levels in the conditional outer kinetochore mutant, stu2-277. STU2 encodes an essential microtubule-associated kinetochore protein that generates force by promoting kinetochore-microtubule depolymerization (He et al. 2001; van Breugel et al. 2003). Mutant stu2-277 cells largely achieve bipolar attachment at the restrictive temperature and arrest in a spindle checkpoint-dependent manner, but fail to undergo normal transient pericentromeric sister separations (He et al. 2001; Pearson et al. 2003; Gillett et al. 2004). Mcd1 ChIPs performed in stu2277 mutant and isogenic wild-type strains revealed that the levels of CHRIII pericentromeric sequences corresponding to a $20 \mathrm{~kb}$ centromere-spanning region were on average 2.4-fold higher in stu2-277 cells than in wildtype cells, while the two strains had equivalent levels of pericentromeric sequences in Mcd1 ChIPs prepared from NZ-treated cells (Fig. 5C). Because stu2 mutants remain bioriented, these results also exclude the possibility that the observed increased cohesin association in the kinetochore mutants is due to increased accessibility of ChIP antibodies to pericentromeric chromatin in the absence of microtubule attachments. 
A

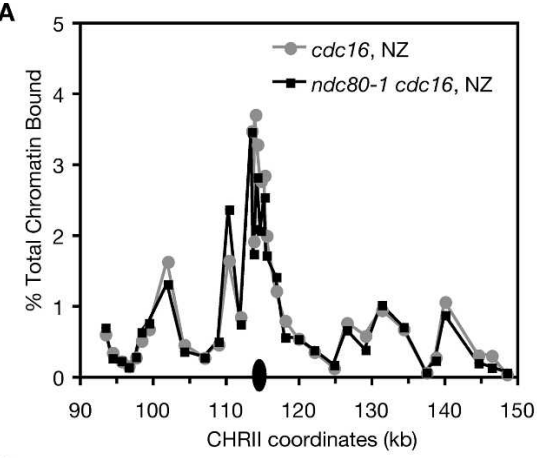

B

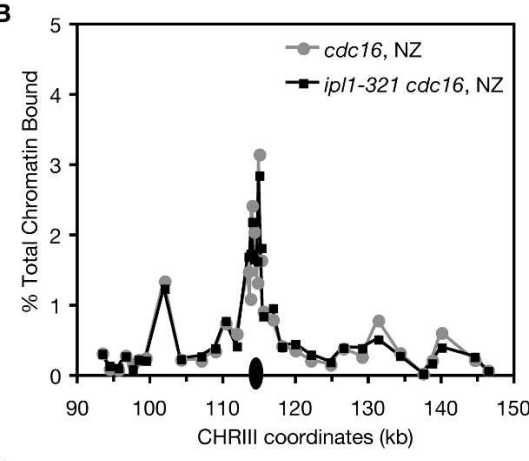

C



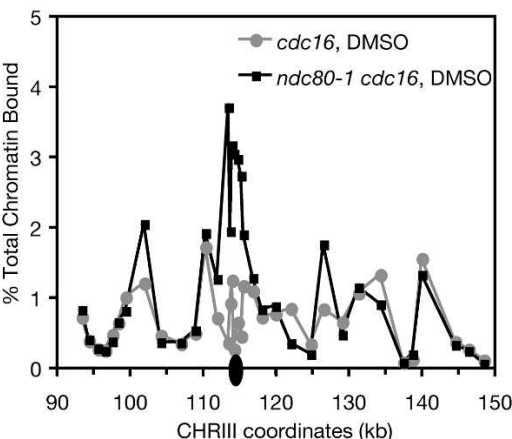


Figure 5. Kinetochore mutants that alter microtubule attachments and/or tension exhibit increased pericentromeric Mcdlp levels. $\mathrm{Mu}$ tant and isogenic controls were staged in G1 using $\alpha$-factor and then released into NZ- or DMSO-containing $37^{\circ} \mathrm{C}$ prewarmed rich media. Cells were processed for ChIP using antibodies against epitope-tagged Mcdl (Mcd1$6 \mathrm{HA}$ or Mcd1-18MYC) when mostly large budded. Metaphase enrichment of cells was confirmed by flow-cytometric analyses (data not shown). Quantitation of CHRIII pericentromeric DNA in Mcd1 ChIPs was performed as described in the legend for Figure 1. $(A)$ Results for Mcd1-18MYC cdc16-123 ip11-321 (1701-38C) or IPL1 (1700-55A) are shown. (B) Results for Mcd1-6HA cdc16-1 ndc80-1 (1849-40D) or NDC80 (1847-22C) are shown. (C) Results for Mcd1-6HA stu2-277 (170419B) or STU2 (1704-18B) are shown.
Our analysis of pericentromeric cohesin association in conditional kinetochore mutants is consistent with the idea that the levels of tension and cohesin association within pericentromeric chromatin are inversely correlated. To extend these observations, we examined cohesin association when pericentromeric tension is relieved without compromising kinetochore integrity. This approach was made possible by placing CDC6, which encodes a DNA replication initiation factor, under the transcriptional control of a galactose-inducible promoter (Materials and Methods). Mono-oriented chromosomes, generated by CDC6 repression in glucose-containing medium, lack tension, but kinetochore-microtubule attachments are sufficiently stable to mediate a reductional anaphase (Piatti et al. 1995). As was the case with the kinetochore mutants, we observed that the levels of CHRIII pericentromeric sequences in Mcd1 ChIPs were 2.5-fold higher throughout an $\sim 20$-kb centromere-flanking region in the strain with unreplicated, mono-oriented chromosomes when compared with the control strain that had replicated, bioriented chromosomes (Fig. 6A). This difference was not due to a defect in cohesin recruitment in unreplicated cells, however, since NZtreated cells that did or did not undergo DNA replication had indistinguishable levels of CHRIII pericentromeric sequences in the Mcd1 ChIPs (Fig. 6B). Taken together, our observations strongly suggest that increased pericentromeric cohesin association is correlated with a reduction of tension across sister kinetochore pairs.

\section{Inner kinetochore subunits mediate enhancer activity}

Our mutational analysis suggested that microtubule-associated proteins, located largely within the outer kinetochore, were not essential for the kinetochore-mediated enhancer activity. We therefore explored the role of a subset of inner and central kinetochore subcomplexes in mediating cohesin recruitment. Homologs of conserved inner kinetochore proteins, CENP-A and CENP$\mathrm{C}$, are present at all functional centromeres in eukaryotes, and are fundamental to kinetochore function (Cleveland et al. 2003). CENP-A is a conserved histone H3 variant that localizes exclusively to centromeric chromatin. Budding yeast CENP-A, known as Cse4, has 
A
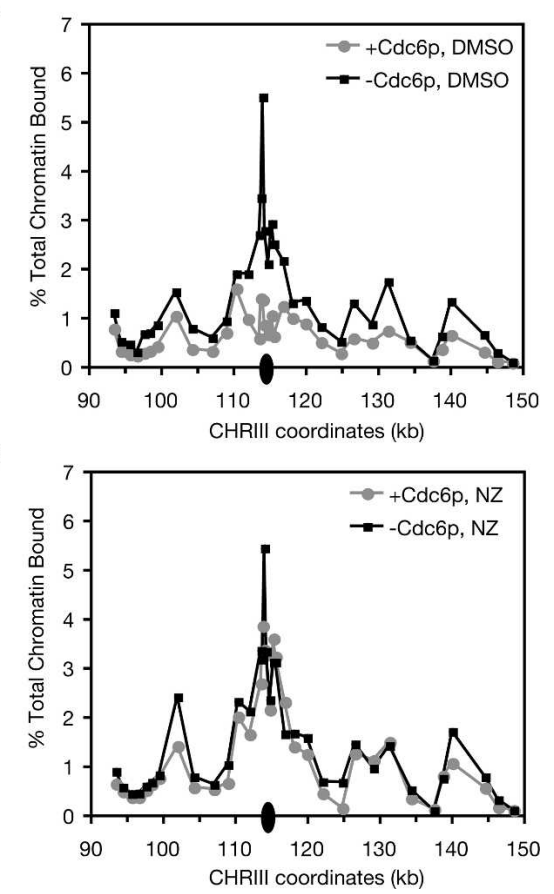

Figure 6. Monopolar attachment results in increased pericentromeric Mcdlp levels. Mcd1-6HA cdc6s pGAL-Ubi-R-CDC6 cells (1705-6A) were grown in rich medium with raffinose and galactose $(2 \%$ each) to allow expression of CDC6. Cells were staged in G1 and released into galactose-containing medium for $20 \mathrm{~min}$ to allow replication to initiate. The culture was then split into two, and one half was washed and resuspended in glucose-containing medium to repress CDC6 expression, while the other half was maintained in galactose-containing medium. Each culture was again treated with $\alpha$-factor to arrest cells at the beginning of the next cell cycle. After G1 arrest, both cultures were released in either $15 \mu \mathrm{g} / \mathrm{mL}$ NZ- or DMSO-containing medium without further change in the medium's carbon source. Cells were collected when mostly large budded, and DNA contents of each culture was confirmed by flow-cytometric analyses (data not shown). (A) The levels of CHRIII pericentromeric sequences in Mcd1 ChIPs in DMSO alone control cells are shown. $(B)$ The levels of CHRIII pericentromeric sequences in Mcd1 ChIPs in NZ-treated cells are shown.

been shown to participate in the recruitment of other kinetochore subcomplexes, such as the COMA complex (Westermann et al. 2003). The stable association of the budding yeast CENP-C homolog, Mif2, with centromeric DNA requires the central kinetochore MIND complex (Pinsky et al. 2003; Westermann et al. 2003), suggesting an elaborate hierarchical assembly of numerous subcomplexes is necessary to form a mature kinetochore.

Conditional kinetochore mutants, cse4-327, mif2-3, MIND complex mutant $m t w 1-1$, and a COMA complex deletion mutant, ctf19s, were analyzed for pericentromeric Mcd1 association. While the other mutants analyzed were spindle checkpoint proficient, the $\operatorname{ctf} 19 \Delta \mathrm{mu}-$ tant was examined in a $c d c 16$ background to generate a uniform mitotic arrest. The levels of CHRIII pericentromeric sequences in Mcd1 ChIPs from each of these mu- tants as well as an isogenic wild-type or cdc16 control were examined in the presence or absence of microtubules. This analysis revealed that all of the mutants had similar levels of CHRIII pericentromeric sequences as their isogenic controls in the presence of microtubules (DMSO) (Fig. 7A-D). In striking contrast, each of the mutants had significantly lower levels of Mcd1 pericentromeric binding than their isogenic control following NZ treatment (Fig. 7A-D). This reduction was greatest, threefold and fourfold, throughout a 20-kb CEN3-spanning region in the $c s e 4-327$ and ctf19s mutants, respectively, while more moderate (twofold) reductions were observed in the mtw1-1 and mif2-3 mutants. These mutants underwent normal cell cycle arrests in response to NZ treatment, suggesting that the low levels of pericentromeric cohesin association were not due to APC-mediated turnover of cohesins. In contrast, cells with a deletion of SLK19, encoding a central kinetochore factor that contributes to the fidelity of kinetochore function, had no difference in pericentromeric cohesin association (Supplementary Fig. 4C; Zhang et al. 2006). Thus, these observations suggest that several inner and central kinetochore subunits play critical roles in the recruitment of elevated levels of pericentromeric cohesin that normally occurs in the absence of tension.

\section{Discussion}

In this study, we provide the first direct evidence that robust pericentromeric cohesion promotes high fidelity chromosome segregation. Previously, it had been impossible to determine the relative contributions of arm and pericentromeric cohesion in chromosome segregation due to the inability to alter cohesion within specific chromosomal regions (Tanaka et al. 2000; Sonoda et al. 2001). However, we have surmounted this technical difficulty by flanking a centromere with "insulator" sequences that limit the kinetochore's ability to mediate pericentromeric cohesin enrichment without affecting arm cohesin association. We observed that the region exhibiting microtubule-dependent stretching is extended in the absence of this enrichment to at least $23 \mathrm{~kb}$ from the centromere, suggesting that $46 \mathrm{~kb}$ of pericentromeric chromatin is deformed rather than the previously estimated $20 \mathrm{~kb}$ in wild-type cells (He et al. 2000). Importantly, we observed an 4000 -fold increase in the frequency of chromosome missegregation in diploids when pericentromeric cohesin association was reduced on CHRIII. This level of chromosome missegregation is significant, and would likely result in inviability if pericentromeric cohesin association were reduced on all chromosomes, rather than on CHRIII alone, as described here. Thus, our results suggest that pericentromeric cohesion plays an important role in the maintenance of genomic integrity that cannot be fully compensated for by remaining arm cohesion. We note that chromosome instability was also observed in fission yeast that had reduced pericentromeric cohesion (Allshire et al. 1995; Nonaka et al. 2002). However, this reduction was mediated by defective heterochromatin formation, making it 

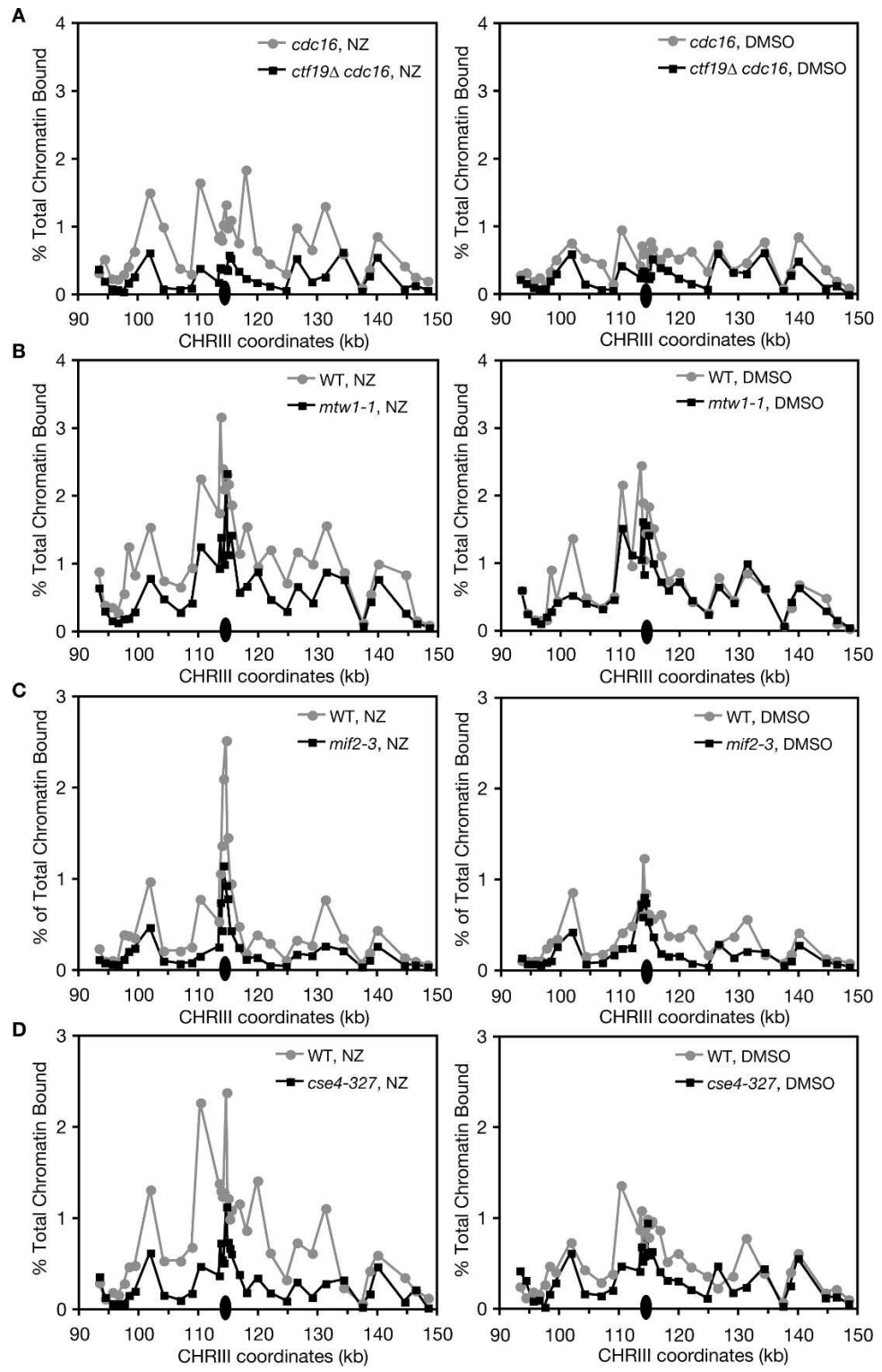

Figure 7. Components of the central and inner kinetochore are required for pericentromeric cohesin enrichment. (A) Mcd1-6HA cdc16-123 ctf19s (CEY57) or isogenic CTF19 (1829-15B) cells were staged in G1 and released into $37^{\circ} \mathrm{C}$ prewarmed media containing either NZ or DMSO. Cells were processed for Mcd1 ChIP when mostly large budded. Cell samples were taken immediately preceding formaldehyde cross-linking for flow-cytometric analyses to confirm metaphase enrichment (data not shown). Analysis of ChIP DNA was done as described in Figure 1. (B) Mcd1-6HA mif2-3 (CEY68) or isogenic MIF2 (CEY67) cells were treated as described in $A$ and processed for Mcdl ChIP. (C) Mcd16HA mtw1-1 (CEY47) or isogenic MTW1 (CEY46) cells were treated as described in $A$ and processed for Mcd1 ChIP. (D) Mcd1-6HA cse4-327 (CEY49) or isogenic CSE4 (CEY46) cells were grown to saturation, diluted into fresh medium, and grown at $37^{\circ} \mathrm{C}$ for $20 \mathrm{~min}$ before addition of NZ or DMSO. difficult to attribute the segregation defect solely to reduced pericentromeric cohesion.

Two models have been proposed to explain the importance of robust pericentromeric cohesion in chromosome biorientation. Dewar et al. (2004) suggest that the sole function of pericentromeric cohesion is to provide tension that stabilizes kinetochore-microtubule attachments. However, our observation that arm cohesion, which remains intact on a chromosome with an insulator-flanked centromere, is incapable of completely compensating for the loss of pericentromeric cohesin enrichment is inconsistent with such a model. An alternative model hypothesizes that robust pericentromeric cohesion imposes a specific geometry in which the kinetochore pairs are held in a back-to-back orientation with the microtubule-binding sites facing opposite poles, thereby promoting amphitelic attachments. Such rigid- ity may be critical in higher eukaryotes to avoid merotely, a condition in which a kinetochore forms attachments to microtubules originating from both poles. Merotely is not possible in budding yeast because each kinetochore forms only one microtubule attachment. However, a geometric model, if correct, predicts an increase in syntelic attachments in budding yeast when pericentromeric cohesion is diminished. We note that overexpression of IPL1, which destablilizes syntelic attachments, partially suppresses the missegregation of a minichromosome containing an insulator-flanked centromere (C.A. Eckert and P.C. Megee, unpubl.). This observation is consistent with the notion that kinetochore geometry may be partially compromised when pericentromeric cohesin association is reduced. In any case, we favor the idea that pericentromeric cohesin enrichment contributes to chromosome biorientation both by con- 
straining sister kinetochores in a favorable orientation for bipolar attachments and also by stabilizing amphitelic attachments.

Our results also demonstrate that the magnitude of pericentromeric cohesin association is sensitive to the status of kinetochore-microtubule attachments. Surprisingly, pericentromeric cohesin association is not increased to counteract poleward microtubule-dependent forces. Rather, we observed that conditional kinetochore mutants that give rise to the complete detachment from microtubules, syntelic attachments, and amphitelic attachments with reduced tension all had elevated levels of pericentromeric, but not arm, cohesin association. Furthermore, pericentromeric cohesin association levels also increased on mono-oriented chromosomes, which lack tension. While the spindle checkpoint is essential for detecting aberrant kinetochore-microtubule attachments, our results indicate that the checkpoint plays no role in the increased pericentromeric cohesin association that accompanies abnormal attachments. Although we cannot exclude the possibility that a novel pathway that monitors pericentromeric tension regulates the kinetochore's enhancer activity for cohesin recruitment, we favor the hypothesis that kinetochore-mediated cohesin recruitment occurs constitutively within pericentromeric chromatin. This model is supported by the observation that pericentromeric cohesin association levels are increased even in mitotically arrested cells following loss of microtubule attachments (P.C. Megee, unpubl.). Thus, pericentromeric cohesin association levels are likely to reflect the balance of two opposing activities: constitutive cohesin recruitment by the kinetochore, and displacement of cohesin binding by the mechanical tension imposed by chromosome biorientation.

The embrace model for sister chromatid cohesion suggests that cohesin topologically encircles replicated sister chromatids (Haering et al. 2002; Gruber et al. 2003). Such an association with chromatin is postulated to allow some flexibility for cohesin localization throughout the genome (Lengronne et al. 2004). Following chromosome biorientation, $\sim 20 \mathrm{~kb}$ of chromatin is deformed $(\mathrm{He}$ et al. 2000, 2001), accompanied by a significant reduction in pericentromeric cohesin association (this study). The imposition of tension is predicted to force cohesin rings to slide laterally away from the kinetochore in the embrace model. However, we find no evidence for an accumulation of cohesin in more centromere-distal locations. Alternatively, the displacement of cohesin from pericentromeric regions may be mediated by the breakage of cohesin rings. While we cannot rule out this possibility, we found that pericentromeric regions of bioriented chromosomes retained cohesin at levels that were typically greater than those observed in at arm cohesinassociated regions. Interestingly, our observation that some cohesin remains bound to stretched chromatin is consistent with a modified topological model in which pericentromeric chromatin under tension assumes a cruciform structure that is held together by intrastrand cohesin associations, rather than an interstrand association (Bloom et al. 2006).
The conservation of cohesin enrichment in eukaryotic pericentromeric regions suggests that robust cohesion flanking kinetochores is important for chromosome segregation. Centromere-associated heterochromatin is important for cohesin recruitment in organisms with regional centromeres, which constitute the vast majority of eukaryotes. Our previous results indicated that heterochromatin-independent mechanisms of pericentromeric cohesin recruitment exist, however, given that budding yeast centromeres lack flanking heterochromatin, but still exhibit pericentromeric cohesin enrichment. We provide evidence here that conserved kinetochore proteins, such as CENP-A and CENP-C, participate in pericentromeric cohesin recruitment. These observations are consistent with previous work showing that CENP-A is required for the association of the Smc1 cohesin complex subunit with centromeric chromatin in humans, and that knockdown of another conserved kinetochore protein, CENP-F, in HeLa cells resulted in weakened pericentromeric cohesion (van Hooser et al. 2001; Holt et al. 2005). Lastly, the loss of pericentromeric heterochromatin in fission yeast did not result in the complete loss of pericentromeric cohesin association, leaving open the possibility that residual levels of cohesin were recruited through a kinetochore-mediated pathway (Nonaka et al. 2002). Thus, our results suggest that mediating pericentromeric cohesin recruitment may be an inherent property of all kinetochores. Such an ability may explain why neocentromeres, which do not have cytologically detectable levels of pericentromeric heterochromatin (Saffery et al. 2000; Amor and Choo 2002), can faithfully mediate chromosome segregation.

Deciphering how the kinetochore mediates the assembly of pericentromeric cohesin-rich domains will be important for a full understanding of the molecular mechanisms of chromosome segregation. Based on our observations, we favor a kinetochore-mediated bidirectional nucleation and spreading mechanism, conceptually similar to those proposed previously for telomeric silencing, dosage compensation, and X-chromosome inactivation, in which cis DNA elements nucleate the assembly of large chromosomal domains (Renauld et al. 1993; Lee et al. 1999; Csankovszki et al. 2004). Such a model is strongly supported by our observation that the flanking of a kinetochore with elements that appear to "insulate" kinetochore enhancer activity significantly reduced cohesin association throughout a large pericentromeric domain (this study). In addition, pericentromeric cohesin domains, established during the preceding $S$ phase, were disassembled in mitotically arrested cells following excision of centromeric DNA, indicating that the kinetochore is also required for the maintenance of pericentromeric cohesin domains (Megee et al. 1999). Precisely how kinetochores mediate this activity is unknown, but may involve the localization of high densities of cohesin loading factors, such as the Scc $2 / \operatorname{Scc} 4$ complex, or histone post-translational modifying factors to pericentromeric chromatin (Lengronne et al. 2004). In any case, the use of these "insulator" elements to regulate cohesin recruitment within specific chromosomal domains will 
enable further dissection of the roles of these domains in mitotic and meiotic chromosome segregation and in DNA repair.

\section{Materials and methods}

\section{Strain construction}

Relevant strain genotypes are listed in Supplementary Table 1. Strains with insulator-flanked CEN3 were constructed as follows. A construct containing CEN3 with flanking CHRIII homology (Weber et al. 2004) was used to introduce a 4.5-kb fragment of Myxococcus GC-rich DNA at a SacI site 37 base pairs (bp) to the left of CEN3 in combination with a 900-bp fragment from the 5' region of PGK1 (CHRIII coordinates 137142-138037) in reverse chromosomal orientation inserted at an XbaI site 79 bp to the right of CEN3. Parental strains with URA3 adjacent to CEN3 were then transformed with the construct and 5-fluoroorotic acid (5-FOA)-resistant transformants were obtained. The loss of URA3 and integration of the insulator-flanked CEN3 construct was confirmed by both PCR and Southern blot analysis of genomic DNA.

\section{Yeast cell culture and cell cycle staging for ChIP}

Unless otherwise indicated, cells used for ChIP were first staged in G1 using $\alpha$-factor mating pheromone, and then released from arrest by washing twice in media containing Pronase (Sigma), as described previously (Weber et al. 2004). Conditional kinetochore mutants and $c d c 16$ strains were released into $37^{\circ} \mathrm{C}$ prewarmed medium. Cultures used for ChIP were either arrested in mitosis using $15 \mu \mathrm{g} / \mathrm{mL} \mathrm{NZ} \mathrm{(Sigma)} \mathrm{in} 1 \%$ DMSO or in $1 \%$ DMSO alone as a vehicle control. Kinetochore mutants that failed to maintain metaphase arrests were analyzed in $c d c 16$ backgrounds to prevent entry into mitosis. Wild-type control strains treated with DMSO that had no means of metaphase arrest were harvested when cells were mostly large budded as determined by light microscopy $(65 \%-90 \%)$, and DNA content was confirmed by flow cytometry to ensure that a mostly metaphase population of cells was present. Mitotic arrest was generally achieved 2-3 h after release from G1.

The cse4-327 mutant (CEY49) is insensitive to $\alpha$-factor (S. Biggins, pers. comm.). Therefore, this strain and an isogenic wild-type control (CEY46) were synchronized by first growing the cells to saturation in rich media. Cells were then diluted to $5 \times 10^{6}$ cells $/ \mathrm{mL}$ in prewarmed media and grown at $37^{\circ} \mathrm{C}$ for 20 min prior to the addition of NZ or DMSO.

CDC6 depletion was done as previously described with minor changes (Biggins and Murray 2001; Stern and Murray 2001). GAL-CDC6 cells (1706-5A), cultured at $30^{\circ} \mathrm{C}$ in rich medium containing raffinose and galactose $(2 \%$ each) were synchronized using $\alpha$-factor. Cells were released from arrest in fresh medium for 20 min to allow DNA replication to initiate, and the culture was then split into two and grown in rich medium with raffinose in the presence or absence of galactose to repress CDC6 expression. After bud emergence ( $40 \mathrm{~min}), \alpha$-factor was again added to these cultures to arrest cells in the next G1. Cells were again released from G1 arrest into fresh media of the same type, but with DMSO alone or NZ. Cells were then processed for ChIP when mostly large budded, and analyzed by flow cytometry to confirm the expected DNA content for each experimental condition (data not shown).

\section{Microscopy}

Lac operator sequences from pCM40 were introduced on the left of the CHRIII pericentromeric region (SGD CHRIII coordinate $\sim 95 \mathrm{~kb}$ ) by transformation of a linear Lac Op plasmid fragment targeted to this region in strains containing either wild-type CEN3 (CEY90) or I-CEN3-I (CEY91) and Lac I-GFP from pCM42 at URA3 (pCM40 and pCM42 were provided by D. Koshland, Carnegie Institution of Washington, Baltimore, MD, unpubl.). Transformants were isolated by selection on $100 \mu \mathrm{g} / \mathrm{mL}$ nourseothricin (clonNAT). Paraformaldehyde-fixed $(4 \%)$ and formaldehyde-fixed $(3.7 \%)$ cells were processed for GFP scoring and indirect immunofluorescence (IF), respectively. Cells $(\geq 100)$ at each time point from two or more independent experiments were scored for bud morphology and number and position of GFP spots. IF was performed using anti-tubulin primary and FITC-conjugated secondary antibodies, and the percentage of anaphase spindles was determined at the indicated intervals $(n=100)$. All scoring was performed under $100 \times$ magnification. FACS analysis was also performed for ethanol-fixed cells at each time point to ensure that timing of two spots corresponded with DNA replication (data not shown).

\section{ChIP}

ChIP was performed as described previously (Megee et al. 1999; Weber et al. 2004). Immunoprecipitations were performed using 12CA5 anti-HA antibody (Roche) or A-14 anti-Myc antibody (Santa Cruz Biotechnology), as indicated. Sequences of PCR primers are available on request. PCR products were resolved on $2.5 \%$ NuSieve (Cambrex) agarose gels containing $0.15 \mu \mathrm{g} / \mathrm{mL}$ ethidium bromide. Digital images of the stained gels were quantitated using ImageQuant software (Molecular Dynamics). ChIP experiments were repeated at least twice, and data from one representative experiment are shown.

\section{Chromosome loss-mating assay}

MATa strains with URA3 inserted at SGD CHRIII coordinate $\sim 303 \mathrm{~kb}$ and either wild-type CEN3 or CEN3 flanked by "insulators" (I-CEN3-I) were mated to a wild-type MAT $\alpha$ strain (18412A) to form diploids CEY39 and CEY61, respectively. A control diploid strain monosomic for the MAT $\alpha$ homolog of CHRIII (CEY102) was obtained by screening 5-FOA-resistant colonies by PCR and Southern blot for those that had lost the CHRIII MATa homolog. For quantitative mating assays, the diploids and control haploids were grown to early exponential phase in rich media (haploids), or in synthetic media lacking uracil (diploids) to ensure that diploids remained disomic for CHRIII before mating. Approximately $5 \times 10^{7}$ cells were mixed with an equal number of cells from MATa and MATa tester strains 1845-36A and 1845-34C, respectively, and plated on rich medium. Because some strains contained bar1 mutations that decreased mating efficiencies, conditioned media containing the Barl protease was used to plate the cells for mating. After $6 \mathrm{~h}$ at $23^{\circ} \mathrm{C}$, mating mixtures were washed from the plates and serial dilutions were spread on selective plates to score the number of mated cells and unmated parent cells. Mating efficiencies were calculated as the percentage of haploid or diploid cells that mated to form diploid or triploid colonies, respectively. Mated colonies were then replica-plated to synthetic media lacking uracil to distinguish chromosome loss from gene conversion events. The reported values are the averages of three independent experiments.

\section{Acknowledgments}

We thank Sue Biggins, Iain Cheeseman, John Kilmartin, Doug Koshland, and Peter Sorger for generously providing strains and plasmids. We thank Judith Jaehning, Doug Koshland, and Robert Sclafani for valuable comments on the manuscript, and Mark Winey and Jennifer Gerton for valuable discussions. This work was supported by the National Institutes of Health, Grant 
R01-GM66213, to P.C.M. C.A.E. acknowledges the support of the Molecular Biology Program and training grant 5T32 GM08730.

\section{References}

Allshire, R.C., Nimmo, E.R., Ekwall, K., Javerzat, J.P., and Cranston, G. 1995. Mutations derepressing silent centromeric domains in fission yeast disrupt chromosome segregation. Genes \& Dev. 9: 218-233.

Amor, D.J. and Choo, K.H. 2002. Neocentromeres: Role in human disease, evolution, and centromere study. Am. J. Hum. Genet. 71: 695-714.

Bernard, P., Maure, J.F., Partridge, J.F., Genier, S., Javerzat, J.P., and Allshire, R.C. 2001. Requirement of heterochromatin for cohesion at centromeres. Science 294: 2539-2542.

Biggins, S. and Murray, A.W. 2001. The budding yeast protein kinase Ipl1/Aurora allows the absence of tension to activate the spindle checkpoint. Genes \& Dev. 15: 3118-3129.

Biggins, S., Severin, F.F., Bhalla, N., Sassoon, I., Hyman, A.A., and Murray, A.W. 1999. The conserved protein kinase Ipl1 regulates microtubule binding to kinetochores in budding yeast. Genes \& Dev. 13: 532-544.

Blat, Y. and Kleckner, N. 1999. Cohesins bind to preferential sites along yeast chromosome III, with differential regulation along arms versus the centric region. Cell 98: 249-259.

Bloom, K. 1993. The centromere frontier: Kinetochore components, microtubule-based motility, and the CEN-value paradox. Cell 73: 621-624.

Bloom, K., Sharma, S., and Dokholyan, N.V. 2006. The path of DNA in the kinetochore. Curr. Biol. 16: R276-R278.

Cheeseman, I.M., Brew, C., Wolyniak, M., Desai, A., Anderson, S., Muster, N., Yates, J.R., Huffaker, T.C., Drubin, D.G., and Barnes, G. 2001a. Implication of a novel multiprotein Dam1p complex in outer kinetochore function. J. Cell Biol. 155: $1137-1145$

Cheeseman, I.M., Enquist-Newman, M., Muller-Reichert, T., Drubin, D.G., and Barnes, G. 2001b. Mitotic spindle integrity and kinetochore function linked by the Duolp/Damlp complex. J. Cell Biol. 152: 197-212.

Cheeseman, I.M., Anderson, S., Jwa, M., Green, E.M., Kang, J., Yates III, J.R., Chan, C.S., Drubin, D.G., and Barnes, G. 2002. Phospho-regulation of kinetochore-microtubule attachments by the Aurora kinase Ipllp. Cell 111: 163-172.

Cleveland, D.W., Mao, Y., and Sullivan, K.F. 2003. Centromeres and kinetochores. From epigenetics to mitotic checkpoint signaling. Cell 112: 407-421.

Cohen-Fix, O. and Koshland, D. 1997. The anaphase inhibitor of Saccharomyces cerevisiae Pdslp is a target of the DNA damage checkpoint pathway. Proc. Natl. Acad. Sci. 94: 1436114366.

Csankovszki, G., McDonel, P., and Meyer, B.J. 2004. Recruitment and spreading of the C. elegans dosage compensation complex along X chromosomes. Science 303: 1182-1185.

Dewar, H., Tanaka, K., Nasmyth, K., and Tanaka, T.U. 2004. Tension between two kinetochores suffices for their bi-orientation on the mitotic spindle. Nature 428: 93-97.

Enquist-Newman, M., Cheeseman, I.M., Van Goor, D., Drubin, D.G., Meluh, P.B., and Barnes, G. 2001. Dad1p, third component of the Duolp/Damlp complex involved in kinetochore function and mitotic spindle integrity. Mol. Biol. Cell 12: $2601-2613$.

Fukagawa, T., Nogami, M., Yoshikawa, M., Ikeno, M., Okazaki, T., Takami, Y., Nakayama, T., and Oshimura, M. 2004 Dicer is essential for formation of the heterochromatin structure in vertebrate cells. Nat. Cell Biol. 6: 784-791.

Gillett, E.S., Espelin, C.W., and Sorger, P.K. 2004. Spindle checkpoint proteins and chromosome-microtubule attachment in budding yeast. J. Cell Biol. 164: 535-546.

Glynn, E.F., Megee, P.C., Yu, H.G., Mistrot, C., Ünal, E., Koshland, D.E., DeRisi, J.L., and Gerton, J.L. 2004. Genome-wide mapping of the cohesin complex in the yeast Saccharomyces cerevisiae. PLoS Biol. 2: 1325-1339.

Goshima, G. and Yanagida, M. 2000. Establishing biorientation occurs with precocious separation of the sister kinetochores, but not the arms, in the early spindle of budding yeast. Cell 100: 619-633.

Gruber, S., Haering, C.H., and Nasmyth, K. 2003. Chromosomal cohesin forms a ring. Cell 112: 765-777.

Haering, C.H., Lowe, J., Hochwagen, A., and Nasmyth, K. 2002. Molecular architecture of SMC proteins and the yeast cohesin complex. Mol. Cell 9: 773-788.

Hardwick, K.G., Weiss, E., Luca, F.C., Winey, M., and Murray, A.W. 1996. Activation of the budding yeast spindle assembly checkpoint without mitotic spindle disruption. Science 273: 953-956.

Hardwick, K.G., Li, R., Mistrot, C., Chen, R.H., Dann, P., Rudner, A., and Murray, A.W. 1999. Lesions in many different spindle components activate the spindle checkpoint in the budding yeast Saccharomyces cerevisiae. Genetics 152: 509_ 518.

Hartman, T., Stead, K., Koshland, D., and Guacci, V. 2000. Pds $5 p$ is an essential chromosomal protein required for both sister chromatid cohesion and condensation in Saccharomyces cerevisiae. J. Cell Biol. 151: 613-626.

He, X., Asthana, S., and Sorger, P.K. 2000. Transient sister chromatid separation and elastic deformation of chromosomes during mitosis in budding yeast. Cell 101: 763-775.

He, X., Rines, D.R., Espelin, C.W., and Sorger, P.K. 2001. Molecular analysis of kinetochore-microtubule attachment in budding yeast. Cell 106: 195-206.

Holt, S.V., Vergnolle, M.A., Hussein, D., Wozniak, M.J., Allan, V.J., and Taylor, S.S. 2005. Silencing CENP-F weakens centromeric cohesion, prevents chromosome alignment and activates the spindle checkpoint. J. Cell Sci. 118: 4889-4900.

Huang, C.E., Milutinovich, M., and Koshland, D. 2005. Rings, bracelet or snaps: Fashionable alternatives for Smc complexes. Philos. Trans. R. Soc. Lond. B Biol. Sci. 360: 537-542.

Ivanov, D. and Nasmyth, K. 2005. A topological interaction between cohesin rings and a circular minichromosome. Cell 122: 849-860.

Janke, C., Ortiz, J., Lechner, J., Shevchenko, A., Magiera, M.M., Schramm, C., and Schiebel, E. 2001. The budding yeast proteins Spc24p and Spc25p interact with Ndc80p and Nuf2p at the kinetochore and are important for kinetochore clustering and checkpoint control. EMBO J. 20: 777-791.

Janke, C., Ortiz, J., Tanaka, T.U., Lechner, J., and Schiebel, E. 2002. Four new subunits of the Dam1-Duol complex reveal novel functions in sister kinetochore biorientation. EMBO J. 21: 181-193.

Kapoor, T.M., Lampson, M.A., Hergert, P., Cameron, L., Cimini, D., Salmon, E.D., McEwen, B.F., and Khodjakov, A. 2006. Chromosomes can congress to the metaphase plate before biorientation. Science 311: 388-391.

Kim, J.H., Kang, J.S., and Chan, C.S. 1999. Sli15 associates with the Ipll protein kinase to promote proper chromosome segregation in Saccharomyces cerevisiae. I. Cell Biol. 145: $1381-1394$.

Laloraya, S., Guacci, V., and Koshland, D. 2000. Chromosomal addresses of the cohesin component Mcd1p. J. Cell Biol. 151: 1047-1056. 
Lee, J.T., Lu, N., and Han, Y. 1999. Genetic analysis of the mouse $\mathrm{X}$ inactivation center defines an $80-\mathrm{kb}$ multifunction domain. Proc. Nat1. Acad. Sci. 96: 3836-3841.

Lengronne, A., Katou, Y., Mori, S., Yokobayashi, S., Kelly, G.P., Itoh, T., Watanabe, Y., Shirahige, K., and Uhlmann, F. 2004. Cohesin relocation from sites of chromosomal loading to places of convergent transcription. Nature 430: 573-578.

Li, Y., Bachant, J., Alcasabas, A.A., Wang, Y., Qin, J., and Elledge, S.J. 2002. The mitotic spindle is required for loading of the DASH complex onto the kinetochore. Genes \& Dev. 16: 183-197.

McCleland, M.L., Gardner, R.D., Kallio, M.J., Daum, J.R., Gorbsky, G.J., Burke, D.J., and Stukenberg, P.T. 2003. The highly conserved Ndc80 complex is required for kinetochore assembly, chromosome congression, and spindle checkpoint activity. Genes \& Dev. 17: 101-114.

Megee, P.C., Mistrot, C., Guacci, V., and Koshland, D. 1999. The centromeric sister chromatid cohesion site directs Mcdlp binding to adjacent sequences. Mol. Cell 4: 445-450.

Miranda, J.J., De Wulf, P., Sorger, P.K., and Harrison, S.C. 2005. The yeast DASH complex forms closed rings on microtubules. Nat. Struct. Mol. Biol. 12: 138-143.

Nasmyth, K. and Haering, C.H. 2005. The structure and function of SMC and kleisin complexes. Annu. Rev. Biochem. 74: 595-648.

Nicklas, R.B. and Ward, S.C. 1994. Elements of error correction in mitosis: Microtubule capture, release, and tension. J. Cell Biol. 126: 1241-1253.

Nonaka, N., Kitajima, T., Yokobayashi, S., Xiao, G., Yamamoto, M., Grewal, S.I., and Watanabe, Y. 2002. Recruitment of cohesin to heterochromatic regions by Swi6/HP1 in fission yeast. Nat. Cell Biol. 4: 89-93.

Pearson, C.G., Maddox, P.S., Zarzar, T.R., Salmon, E.D., and Bloom, K. 2003. Yeast kinetochores do not stabilize Stu2pdependent spindle microtubule dynamics. Mol. Biol. Cell 14: 4181-4195.

Piatti, S., Lengauer, C., and Nasmyth, K. 1995. Cdc6 is an unstable protein whose de novo synthesis in G1 is important for the onset of S phase and for preventing a 'reductional' anaphase in the budding yeast Saccharomyces cerevisiae. EMBO J. 14: 3788-3799.

Pinsky, B.A. and Biggins, S. 2005. The spindle checkpoint: Tension versus attachment. Trends Cell Biol. 15: 486-493.

Pinsky, B.A., Tatsutani, S.Y., Collins, K.A., and Biggins, S. 2003. An Mtw1 complex promotes kinetochore biorientation that is monitored by the Ipl1/Aurora protein kinase. Dev. Cell 5: 735-745.

Pinsky, B.A., Kung, C., Shokat, K.M., and Biggins, S. 2006. The Ipl1-Aurora protein kinase activates the spindle checkpoint by creating unattached kinetochores. Nat. Cell Biol. 8: 7883.

Renauld, H., Aparicio, O.M., Zierath, P.D., Billington, B.L., Chhablani, S.K., and Gottschling, D.E. 1993. Silent domains are assembled continuously from the telomere and are defined by promoter distance and strength, and by SIR3 dosage. Genes \& Dev. 7: 1133-1145.

Saffery, R., Irvine, D.V., Griffiths, B., Kalitsis, P., Wordeman, L., and Choo, K.H. 2000. Human centromeres and neocentromeres show identical distribution patterns of $>20$ functionally important kinetochore-associated proteins. Hum. Mol. Genet. 9: 175-185.

Skibbens, R.V., Skeen, V.P., and Salmon, E.D. 1993. Directional instability of kinetochore motility during chromosome congression and segregation in mitotic newt lung cells: A pushpull mechanism. J. Cell Biol. 122: 859-875.

Sonoda, E., Matsusaka, T., Morrison, C., Vagnarelli, P., Hoshi,
O., Ushiki, T., Nojima, K., Fukagawa, T., Waizenegger, I.C., Peters, J.M., et al. 2001. Scc1/Rad21/Mcd1 is required for sister chromatid cohesion and kinetochore function in vertebrate cells. Dev. Cell 1: 759-770.

Stern, B.M. and Murray, A.W. 2001. Lack of tension at kinetochores activates the spindle checkpoint in budding yeast. Curr. Biol. 11: 1462-1467.

Tanaka, T., Fuchs, J., Loidl, J., and Nasmyth, K. 2000. Cohesin ensures bipolar attachment of microtubules to sister centromeres and resists their precocious separation. Nat. Cell Biol. 2: 492-499.

Tanaka, T.U., Rachidi, N., Janke, C., Pereira, G., Galova, M., Schiebel, E., Stark, M.J., and Nasmyth, K. 2002. Evidence that the Ipl1-Sli15 (Aurora kinase-INCENP) complex promotes chromosome bi-orientation by altering kinetochorespindle pole connections. Cell 108: 317-329.

Ünal, E., Arbel-Eden, A., Sattler, U., Shroff, R., Lichten, M., Haber, J.E., and Koshland, D. 2004. DNA damage response pathway uses histone modification to assemble a doublestrand break-specific cohesin domain. Mol. Cell 16: 991-1002.

van Breugel, M., Drechsel, D., and Hyman, A. 2003. Stu2p, the budding yeast member of the conserved Dis1/XMAP215 family of microtubule-associated proteins is a plus end-binding microtubule destabilizer. J. Cell Biol. 161: 359-369.

van Hooser, A.A., Ouspenski, I.I., Gregson, H.C., Starr, D.A., Yen, T.J., Goldberg, M.L., Yokomori, K., Earnshaw, W.C., Sullivan, K.F., and Brinkley, B.R. 2001. Specification of kinetochore-forming chromatin by the histone $\mathrm{H} 3$ variant CENP-A. J. Cell Sci. 114: 3529-3542.

Waters, J.C., Skibbens, R.V., and Salmon, E.D. 1996. Oscillating mitotic newt lung cell kinetochores are, on average, under tension and rarely push. J. Cell Sci. 109: 2823-2831.

Weber, S.A., Gerton, J.L., Polancic, J.E., DeRisi, J.L., Koshland, D., and Megee, P.C. 2004. The kinetochore is an enhancer of pericentric cohesin binding. PLOS Biol. 2: 1340-1353.

Westermann, S., Cheeseman, I.M., Anderson, S., Yates III, J.R., Drubin, D.G., and Barnes, G. 2003. Architecture of the budding yeast kinetochore reveals a conserved molecular core. $J$. Cell Biol. 163: 215-222.

Wigge, P.A. and Kilmartin, J.V. 2001. The Ndc80p complex from Saccharomyces cerevisiae contains conserved centromere components and has a function in chromosome segregation. J. Cell Biol. 152: 349-360.

Yamamoto, A., Guacci, V., and Koshland, D. 1996. Pds1p, an inhibitor of anaphase in budding yeast, plays a critical role in the APC and checkpoint pathway(s). I. Cell Biol. 133: 99110.

Zhang, T., Lim, H.H., Cheng, C.S., and Surana, U. 2006. Deficiency of centromere-associated protein Slk19 causes premature nuclear migration and loss of centromeric elasticity. $J$. Cell Sci. 119: 519-531. 




\title{
The enhancement of pericentromeric cohesin association by conserved kinetochore components promotes high-fidelity chromosome segregation and is sensitive to microtubule-based tension
}

\author{
Carrie A. Eckert, Daniel J. Gravdahl and Paul C. Megee
}

Genes Dev. 2007, 21: originally published online January 22, 2007

Access the most recent version at doi:10.1101/gad.1498707

$\begin{array}{cl}\begin{array}{c}\text { Supplemental } \\ \text { Material }\end{array} & \text { http://genesdev.cshlp.org/content/suppl/2007/01/22/gad.1498707.DC1 } \\ \text { Related Content } & \begin{array}{l}\text { Enhancing togetherness: kinetochores and cohesion } \\ \text { Jennifer L. Gerton } \\ \text { Genes Dev. February, 2007 21:238-241 }\end{array} \\ \text { References } & \begin{array}{l}\text { This article cites } 64 \text { articles, } 33 \text { of which can be accessed free at: } \\ \text { http://genesdev.cshlp.org/content/21/3/278.full.html\#ref-list-1 }\end{array} \\ & \begin{array}{l}\text { Articles cited in: } \\ \text { http://genesdev.cshlp.org/content/21/3/278.full.html\#related-urls }\end{array} \\ \text { License } & \begin{array}{l}\text { Receive free email alerts when new articles cite this article - sign up in the box at the top } \\ \text { right corner of the article or click here. }\end{array}\end{array}$

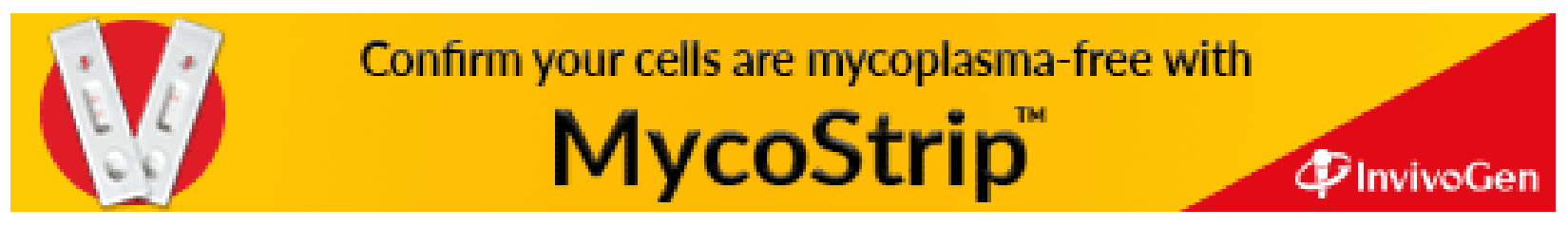

